\title{
Long Baseline GPS+BDS RTK Positioning with Partial Ambiguity Resolution
}

\author{
Andreas Brack \\ Institute for Communications and Navigation \\ Technische Universität München (TUM), Munich, Germany
}

\section{BIOGRAPHY}

Andreas Brack received the B.Sc. and M.Sc. degrees in electrical engineering from Technische Universität München (TUM), Munich, Germany, in 2010 and 2012, respectively. He is currently a Ph.D. candidate at the Institute for Communications and Navigation, TUM. His research focuses on ambiguity resolution techniques for future high-precision GNSS applications.

\begin{abstract}
Reliable real-time kinematic positioning requires the occurrence of incorrect integer ambiguity estimates to be limited to a maximum tolerable rate. For long baselines this usually implies long convergence times due to the presence of atmospheric delay parameters. Several simulation studies have shown that partial ambiguity resolution (PAR) techniques are beneficial in terms of faster solutions, since it is more likely that a subset of all ambiguities can be reliably resolved rather than the full set, but they also result in a positioning precision that is inferior to the one after successful full ambiguity resolution. We analyze the impact of PAR on the positioning capabilities of dual-frequency single and combined GPS and BDS on a long baseline. It will be demonstrated in numerical simulations that the time to reach centimeter level positioning results can be expected to be clearly reduced when using PAR techniques, in particular for the combined system. This will be verified with one day of real global navigation satellite system data from an $88.5 \mathrm{~km}$ baseline in the area of Perth, Australia.
\end{abstract}

\section{INTRODUCTION}

The benefit of using partial ambiguity resolution (PAR) techniques for single long baseline real-time kinematic (RTK) positioning is analyzed and verified with real global navigation satellite system (GNSS) data. With long baseline it is referred to the case that the spatial correlation between the atmospheric delays experienced by the signals observed at the two receivers is too weak to be properly utilized. The residual between receiver differential tropospheric zenith wet delay and the double difference ionospheric slant delays are thus included as unknowns in the parameter vector and estimated along with the user coordinates and the carrierphase integer ambiguities. This is the so-called atmosphere float model. The RTK capabilities are limited by the need for correct estimates of the carrier-phase integer ambiguities, since incorrect ambiguity estimates can lead to large errors in the estimated user position. Ideally, the ambiguity resolution scheme is designed such that a user-defined maximum tolerable rate of an incorrect integer estimate is not exceeded [1,2].

This failure rate constraint generally leads to long convergence times for the atmosphere float model, i.e., many epochs of measurement data have to be collected before the carrier-phase ambiguities can be reliably resolved and the ambiguity fixed coordinate precision is reached. In such cases it is beneficial to consider PAR techniques, which resolve only a subset of ambiguities. They allow for faster solutions, since it is more likely that a subset of all ambiguities can be reliably resolved rather than the full set. However, one has to keep in mind that the resulting positioning precision is generally lower than the one after successful full ambiguity resolution (FAR), since the integer property of the ambiguities is not fully exploited. It was demonstrated via numerical studies that PAR techniques can clearly shorten the time to reach centimeter level accuracy for long baselines $[3,4,5]$.

Improved ambiguity resolution performance can be achieved by combining multiple GNSS, as it was shown for GPS and Galileo [6], GPS and BDS [7, 8, 9], and four system GPS, Galileo, BDS, and QZSS [10]. Instantaneous and reliable ambiguity resolution for long baselines, however, is not possible [6] and fast solutions still remain difficult [11, 12], even in such a combined GNSS case. The real data results for instantaneous GPS+BDS RTK positioning in [13] show that PAR is particularly useful for a combined system. The capabilities of PAR for GPS+BDS positioning on a long baseline are investigated in this contribution.

There are two fundamentally different strategies to approach the problem of reliable FAR or PAR, i.e., to determine the subset of ambiguities to be resolved and the corresponding integer solution. Model-driven approaches only make use of the underlying 
system model, whereas the more elaborate but also more flexible data-driven approaches also utilize the measurement data itself. The performance of each a model-driven and a data-driven algorithm for FAR and PAR is analyzed for dual-frequency single and combined GPS and BDS positioning on an $88.5 \mathrm{~km}$ baseline in the area of Perth, Australia. It will be shown that the PAR schemes can be expected to clearly reduce the average time required to reach centimeter level positioning results compared to the conventional FAR schemes, especially for the combined system. This will be verified using one day of real GNSS data collected at the two IGS MGEX stations PERT and NNOR.

\section{RELIABLE RTK POSITIONING}

The system of linearized GNSS observation equations for long baseline positioning can be written in the form

$$
\boldsymbol{y}=\boldsymbol{A} \boldsymbol{a}+\boldsymbol{B b}+\boldsymbol{\eta}
$$

where $\boldsymbol{y} \in \mathbb{R}^{q}$ contains the double difference code and carrier-phase observations of all previous epochs, $\boldsymbol{a} \in \mathbb{Z}^{n}$ the time constant carrier-phase integer ambiguities, and $\boldsymbol{b} \in \mathbb{R}^{p}$ the incremental baseline coordinates, the residual tropospheric zenith delay, and the double difference ionospheric slant delays for each measurement epoch. The noise vector $\boldsymbol{\eta} \in \mathbb{R}^{q}$ is assumed to follow a zero mean Gaussian distribution and to properly model the correlations induced by double differencing. Dynamic models linking the atmospheric parameters from different epochs may be included in (1).

The estimation of $\boldsymbol{b}$ can be decomposed into three steps. The first one is the Gaussian distributed so-called float solution $\hat{\boldsymbol{a}}$ and $\hat{b}$ from a linear least-squares estimation

$$
\left[\begin{array}{l}
\hat{a} \\
\hat{b}
\end{array}\right] \sim \mathcal{N}\left(\left[\begin{array}{l}
a \\
b
\end{array}\right],\left[\begin{array}{cc}
Q_{\hat{a}} & Q_{\hat{a} \hat{b}} \\
Q_{\hat{b} \hat{a}} & Q_{\hat{b}}
\end{array}\right]\right)
$$

The second step is the integer ambiguity resolution, which is based on the covariance matrix $\boldsymbol{Q}_{\hat{\boldsymbol{a}}}$ and the realization of $\hat{\boldsymbol{a}}$. The integer mapping takes place either in the original $n$-dimensional ambiguity space or after the transformation

$$
\hat{z}=Z \hat{\boldsymbol{a}} \quad \text { and } \quad Q_{\hat{z}}=Z Q_{\hat{a}} Z^{\mathrm{T}}
$$

where $\boldsymbol{Z} \in \mathbb{Z}^{n \times n}$ is an integer unimodular matrix with $|\operatorname{det}(\boldsymbol{Z})|=1$. Such a transformation may or may not affect the results for FAR algorithms, but it fundamentally changes the problem of PAR, irrespective of the integer mapping that is used. As will be shown later, decorrelation transformations [14] prove to be very useful for PAR. After the parameterization of the ambiguities via $Z$ has been chosen, some (PAR) or all (FAR) of the ambiguities may be fixed to integers. Let the set of indexes that correspond to the ambiguities that are resolved and kept as float values be given by $\mathcal{I}$ and $\overline{\mathcal{I}}$, respectively (with $\mathcal{I} \subseteq\{1, \ldots, n\}$, $\mathcal{I} \cup \overline{\mathcal{I}}=\{1, \ldots, n\}$, and $\mathcal{I} \cap \overline{\mathcal{I}}=\emptyset$ ). If the selection of the subset $\mathcal{I}$ is based only on $\boldsymbol{Q}_{\hat{z}}$, we call this a purely model-driven scheme, whereas if both $\boldsymbol{Q}_{\hat{z}}$ and the realization of $\hat{z}$ are used, we refer to this as a data-driven approach. All ambiguities with an index in $\mathcal{I}$ are fixed to integers

$$
\check{z}=\mathcal{S}(\hat{z}), \quad \mathcal{S}(\cdot): \mathbb{R}^{n} \mapsto \mathbb{Z}^{|\mathcal{I}|} .
$$

Note that the fixed solution $\check{z}$ is of dimension $|\mathcal{I}|$ instead of $n$. For reliable coordinate estimates, the mapping $\mathcal{S}(\cdot)$ has to be chosen such that the probability of an incorrect ambiguity solution $P\left(\check{\boldsymbol{z}} \neq \boldsymbol{z}_{\mathcal{I}}\right)$ does not exceed the predefined value $P_{\mathrm{f}}$, with $\boldsymbol{z}=\boldsymbol{Z} \boldsymbol{a}$. The properties of admissible integer mappings $\mathcal{S}(\cdot)$ are formulated in the context of integer aperture estimation [15] for FAR and generalized integer aperture estimation [16] for PAR. Four realizations of $\mathcal{S}(\cdot)$ are presented later in this section.

In the third and final step, the estimates of the real valued parameters $\hat{b}$ are refined by conditioning them to the fixed ambiguities

$$
\check{\boldsymbol{b}}=\hat{\boldsymbol{b}}-\boldsymbol{Q}_{\hat{\boldsymbol{b}} \hat{\boldsymbol{z}}_{\mathcal{I}}} \boldsymbol{Q}_{\hat{\boldsymbol{z}}_{\mathcal{I}}}^{-1}\left(\hat{\boldsymbol{z}}_{\mathcal{I}}-\check{\boldsymbol{z}}\right),
$$

with $\boldsymbol{Q}_{\hat{b} \hat{\boldsymbol{z}}_{\mathcal{I}}}=\boldsymbol{Q}_{\hat{\boldsymbol{b}} \hat{\boldsymbol{a}}} \boldsymbol{Z}_{\mathcal{I}}^{\mathrm{T}}$ and $\boldsymbol{Q}_{\hat{\boldsymbol{z}}_{\mathcal{I}}}=\boldsymbol{Z}_{\mathcal{I}} \boldsymbol{Q}_{\hat{\boldsymbol{a}}} \boldsymbol{Z}_{\mathcal{I}}^{\mathrm{T}}$. The partially ambiguity fixed precision of the estimates of $\boldsymbol{b}$ for a given specific index set $\mathcal{I}=\mathcal{J}$ (note that $\mathcal{I}$ is a discrete random variable for data-driven fixing schemes) is described by the conditional covariance matrix

$$
\boldsymbol{Q}_{\hat{\boldsymbol{b}} \mid \hat{\boldsymbol{z}}_{\mathcal{J}}}=\boldsymbol{Q}_{\hat{\boldsymbol{b}}}-\boldsymbol{Q}_{\hat{\boldsymbol{b}}_{\hat{\boldsymbol{J}}}} \boldsymbol{Q}_{\hat{\boldsymbol{z}}_{\mathcal{J}}}^{-1} \boldsymbol{Q}_{\hat{\boldsymbol{z}}_{\mathcal{J}} \hat{\boldsymbol{b}}}
$$

Given that the ambiguity failure rate is sufficiently small, the user can make use of (6) to compute the precision that he can expect from the coordinate estimates once the index set has been determined by the employed algorithm. 


\section{Reliable Model-driven Ambiguity Resolution}

For model-driven algorithms one specific index set $\mathcal{I}$ has to be selected based on $\boldsymbol{Q}_{\hat{\boldsymbol{z}}}$, such that the probability of a wrong ambiguity estimate does not exceed $P_{\mathrm{f}}$, if this specific subset of ambiguities is resolved irrespective of the realization of $\hat{\boldsymbol{z}}$.

For FAR, the index set $\mathcal{I}$ can only assume the two values $\mathcal{I}=\{1, \ldots, n\}$ or $\mathcal{I}=\emptyset$, i.e., either the full set of ambiguities is resolved or none of them is. Integer least-squares (ILS) is optimal in the sense of maximizing the probability of correct integer estimates for $\mathcal{I}=\{1, \ldots, n\}$ and is thus most likely to meet the failure rate constraint. Its failure rate cannot be computed in closed form, but an upper bound is given by the integer bootstrapping (IB) failure rate [17]

$$
P_{\mathrm{f}, \mathrm{IB}}=1-\prod_{i=1}^{n}\left(2 \Phi\left(\frac{1}{2 \sigma_{\hat{z}_{i \mid I}}}\right)-1\right),
$$

with $\Phi(\cdot)$ the cumulative distribution function of the standard Gaussian, $\sigma_{\hat{z}_{i \mid I}}$ the conditional standard deviation of the transformed ambiguity from $\boldsymbol{Q}_{\hat{z}}$, and $I=\{i+1, \ldots, n\}$. The IB failure rate is a sharp upper bound if a prior decorrelation is applied $[18,19]$. In the following section, IB FAR refers to an evaluation of $P_{\mathrm{f}, \mathrm{IB}}$, followed by $\mathrm{ILS}$ if $P_{\mathrm{f}, \mathrm{IB}} \leq P_{\mathrm{f}}$.

For PAR, the index set $\mathcal{I}$ can assume any of the $2^{n}$ possibilities that result from either including each of the $n$ ambiguities in $\mathcal{I}$ or not. The optimal estimator for a given subset $\mathcal{I}$ leading to the highest possible probability of correct estimates is discussed in [20] and given by

$$
\check{\boldsymbol{z}}=\underset{\boldsymbol{u} \in \mathbb{Z}^{|\mathcal{I}|}}{\operatorname{argmax}} \sum_{\boldsymbol{v} \in \mathbb{Z}^{n} \mid \boldsymbol{v}_{\mathcal{I}}=\boldsymbol{u}} \exp \left(-\frac{1}{2}\|\hat{\boldsymbol{z}}-\boldsymbol{v}\|_{\boldsymbol{Q}_{\hat{\boldsymbol{z}}}}^{2}\right) .
$$

An upper bound of its failure rate is given by the IB failure rate corresponding to the partial covariance matrix $\boldsymbol{Q}_{\hat{\boldsymbol{z}}_{\mathcal{I}}}$. In the following section, IB PAR refers to (8) combined with the very simple subset selection strategy $\mathcal{I}=\left\{n_{\mathrm{IB}}, \ldots, n\right\}$, with

$$
n_{\mathrm{IB}}=\underset{n^{\prime} \in\{1, \ldots, n\}}{\operatorname{argmax}} P_{\mathrm{f}, \mathrm{IB}, n^{\prime}} \quad \text { s.t. } \quad P_{\mathrm{f}, \mathrm{IB}, n^{\prime}} \leq P_{\mathrm{f}},
$$

where the partial IB failure rate $P_{\mathrm{f}, \mathrm{IB}, n^{\prime}}$ is given by

$$
P_{\mathrm{f}, \mathrm{IB}, n^{\prime}}=1-\prod_{i=n^{\prime}}^{n}\left(2 \Phi\left(\frac{1}{2 \sigma_{\hat{z}_{i \mid I}}}\right)-1\right) .
$$

This idea of using a truncated version of IB for reliable PAR was introduced in [21] and used in, e.g., [3, 22, 23].

\section{Reliable Data-driven Ambiguity Resolution}

For data-driven algorithms the selection of the index set $\mathcal{I}$ depends on both $\boldsymbol{Q}_{\hat{z}}$ and the realization of $\hat{z}$ itself, i.e., $\mathcal{I}$ is now a random variable assuming discrete values. The estimator has to be designed such that the overall probability of an incorrect integer solution does not exceed $P_{\mathrm{f}}$.

For FAR this means that only if the float solution $\hat{z}$ falls into a certain subset of $\mathbb{R}^{n}$, which is referred to as the aperture region [15], all $n$ ambiguities are resolved, otherwise none of them is. In practice, the aperture region is often implicitly defined via an acceptance test of the ILS solution $\boldsymbol{z}^{*}=\operatorname{argmin}_{\boldsymbol{v} \in \mathbb{Z}^{n}}\|\hat{\boldsymbol{z}}-\boldsymbol{v}\|_{\boldsymbol{Q}_{\hat{\boldsymbol{z}}}}^{2}$ against the second best solution $\overline{\boldsymbol{z}}=\operatorname{argmin}_{\boldsymbol{v} \in \mathbb{Z}^{n} \backslash \boldsymbol{z}^{*}}\|\hat{\boldsymbol{z}}-\boldsymbol{v}\|_{\boldsymbol{Q}_{\hat{\boldsymbol{z}}}}^{2}$. A popular test of that form is the difference test (DT) [24]

$$
\mathcal{I}= \begin{cases}\{1, \ldots, n\} & \text { if }\|\hat{\boldsymbol{z}}-\overline{\boldsymbol{z}}\|_{\boldsymbol{Q}_{\hat{\boldsymbol{z}}}}^{2}-\left\|\hat{\boldsymbol{z}}-\boldsymbol{z}^{*}\right\|_{\boldsymbol{Q}_{\hat{\boldsymbol{z}}}}^{2} \geq \mu \\ \emptyset & \text { else. }\end{cases}
$$

By properly adjusting the critical value $\mu$, a fixed failure rate $P_{\mathrm{f}}$ can be guaranteed.

The theory of integer aperture estimation can be extended to PAR by introducing at most $2^{n}$ generalized aperture regions, where each one is linked to one specific realization of the index set $\mathcal{I}$ [16]. A possible strategy for defining these regions is to not only test the best integer solution $z^{*}$ against the second best solution, but against the best counter-hypothesis $\bar{z}^{i}=$ $\operatorname{argmin}_{\boldsymbol{v} \in \mathbb{Z}^{n} \mid \boldsymbol{v}_{i} \neq \boldsymbol{z}_{i}^{*}}\|\hat{\boldsymbol{z}}-\boldsymbol{v}\|_{\boldsymbol{Q}_{\hat{\boldsymbol{z}}}}^{2}$ for each scalar ambiguity. The modified version of the DT follows as

$$
\mathcal{I}=\left\{i=1, \ldots, n \mid\left\|\hat{z}-\overline{\boldsymbol{z}}^{i}\right\|_{\boldsymbol{Q}_{\hat{\boldsymbol{z}}}}^{2}-\left\|\hat{\boldsymbol{z}}-\boldsymbol{z}^{*}\right\|_{\boldsymbol{Q}_{\hat{\boldsymbol{z}}}}^{2} \geq \mu\right\}
$$

Again, a fixed failure rate can be guaranteed by properly choosing the critical value $\mu$. The integer solution for this strategy is given by $\check{z}=z_{\mathcal{I}}^{*}$. It is essential for this scheme to be applied in a decorrelated ambiguity space. Otherwise, many or even all of the counter-hypotheses $\overline{\boldsymbol{z}}^{i}$ are likely to be identical and PAR would become FAR. 


\section{EXPERIMENTAL RESULTS}

The performance of the four above introduced ambiguity resolution algorithms is analyzed for dual-frequency long baseline single and combined GPS and BDS positioning with simulations and for combined GPS+BDS also with real GNSS data. The real data was collected at the two IGS MGEX stations PERT (Trimble NetR9) and NNOR (Septentrio PolaRx4) in the area of Perth, Australia, during September 7, 2015. The stations form a baseline with a length of $88.5 \mathrm{~km}$. The number of visible satellites with an elevation angle greater than $10^{\circ}$ is shown in Figure 1. The GPS and BDS signals that are used are listed in Table 1 together with the assumed zenith referenced standard deviations of the measurement noise, to which the elevation dependent exponential weighting function from [25] is applied. The code standard deviations were estimated from one day of double difference code measurements recorded on a short baseline by receivers of similar type with known coordinates, see [13]. Since GPS and BDS do not share common frequencies, a separate pivot satellite is used for both systems. The measurements are a-priori corrected for the hydrostatic tropospheric delays using the blind MOPS tropospheric model [26] and the Niell mapping function [27].

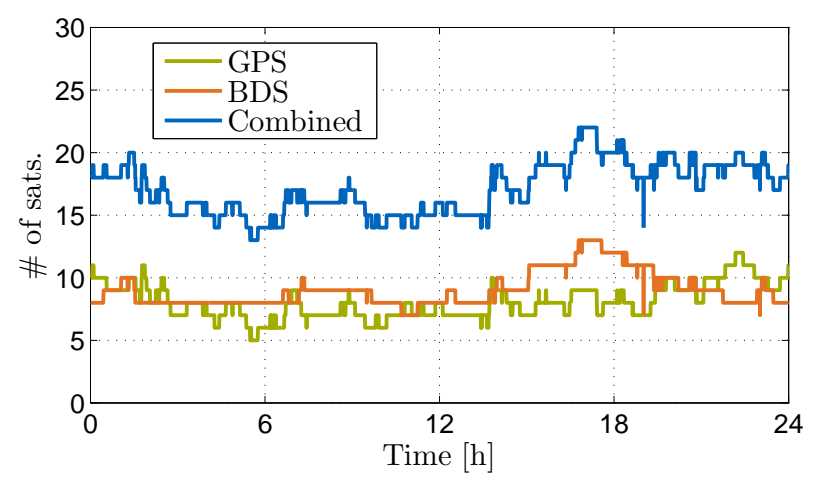

Figure 1: Number of visible GPS and BDS satellites during September 7, 2015.

Table 1: Wavelengths and zenith referenced code and carrier-phase standard deviations of GPS and BDS observables.

\begin{tabular}{|l|cccc|}
\hline & L1 & L2 & B1 & B2 \\
\hline$\lambda[\mathrm{cm}]$ & 19.03 & 24.42 & 19.20 & 24.83 \\
$\sigma_{\rho}[\mathrm{cm}]$ & 37 & 28 & 35 & 28 \\
$\sigma_{\varphi}[\mathrm{mm}]$ & 2 & 2 & 2 & 2 \\
\hline
\end{tabular}

The float solutions are computed using a recursive least-squares implementation, in which the ambiguities are assumed time constant. The relative tropospheric wet zenith delay is modeled as a random walk with a process noise of $2 \mathrm{~mm} / \sqrt{\mathrm{hour}}$, and the coordinates and ionospheric delays are assumed completely unlinked in time. No correlations are assumed between the measurements from consecutive epochs. Rising satellites are included immediately in the computation of the float solution. The ambiguity resolution algorithms are applied anew in each epoch, i.e., the ambiguities are not held fixed once an integer solution has been found.

The computation of the IB failure rate for IB FAR is carried out after applying the LAMBDA decorrelation [28], and the two PAR schemes are applied in the LAMBDA decorrelated ambiguity space. The model dependent critical values for the data-driven DT based schemes are approximated with the conservative functional description from [29]. The maximum failure rate is set to $P_{\mathrm{f}}=0.1 \%$. In order to visualize the computed formal precision as given by $\boldsymbol{Q}_{\hat{\boldsymbol{b}} \mid \hat{\boldsymbol{z}}}$ in (6), we define the scalar formal precision measure

$$
\alpha=\max \left\{\frac{\sigma_{\mathrm{E}}}{1 \mathrm{~cm}}, \frac{\sigma_{\mathrm{N}}}{1 \mathrm{~cm}}, \frac{\sigma_{\mathrm{U}}}{3 \mathrm{~cm}}\right\}
$$

where $\sigma_{\mathrm{E}}, \sigma_{\mathrm{N}}$, and $\sigma_{\mathrm{U}}$ are the standard deviations of the coordinate estimates in the local east, north, and up frame that follow from $\boldsymbol{Q}_{\hat{\boldsymbol{b}} \mid \hat{\boldsymbol{z}}_{\mathcal{J}}}$.

\section{Predicted Times to Reach Centimeter Level Positioning Results}

We define the convergence time as the number of epochs required with IB FAR to reach a formal precision of $\alpha=2$, i.e., a standard deviation of $2 \mathrm{~cm}$ in the horizontal plane, or $\alpha=10$, i.e., decimeter level precision in the horizontal plane. The average 
Table 2: Average convergence times of IB FAR in terms of the required number of epochs; one epoch corresponds to $30 \mathrm{~s}$.

\begin{tabular}{|l|cc|}
\hline & $\alpha=2$ & $\alpha=10$ \\
\hline GPS L1 L2 & 58.7 & 52.3 \\
BDS B1 B2 & 45.1 & 44.3 \\
GPS L1 L2 + BDS B1 B2 & 26.2 & 21.0 \\
\hline
\end{tabular}

convergence times are given in Table 2, where the estimation is started every 10 min throughout the day. The smaller values for $\alpha=10$ imply that this precision may already be reached with the float solution. Improved convergence times are obtained with the combined system.

Since the model-driven IB FAR is always the weakest scheme, it is now investigated via simulations by how much the convergence times can be expected to be reduced on average by using one of the more advanced ambiguity resolution schemes. Figure 2 shows the average availability of precise and reliable coordinate estimates as a function of the fraction of the convergence time of IB FAR, where precise means $\alpha=2$ in the first row and $\alpha=10$ in the second row. For the combined system we can see that by using IB PAR the time required to reach $\alpha=2$ can be reduced by half in $50 \%$ of the cases. Similar results are obtained when using the data-driven DT FAR instead of IB FAR. The data-driven DT PAR scheme can even reduce the time to reach $\alpha=2$ by half with an average probability of more than $95 \%$ and by $75 \%$ with an average probability of $\sim 50 \%$. That is, we can expect that the PAR schemes lead to clearly faster solutions compared to their FAR counterparts.
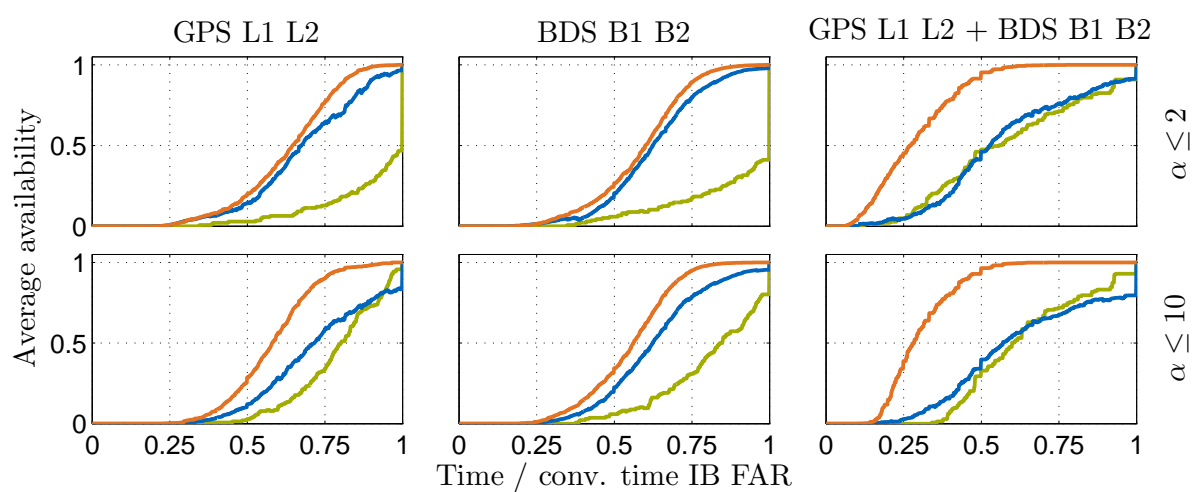

Figure 2: Average reduction of time required to reach $\alpha=2$ (first row) and $\alpha=10$ (second row); fixed failure rate $P_{\mathrm{f}}=0.1 \%$; green: IB PAR, blue: DT FAR, orange: DT PAR.

\section{Real Data Analysis}

The positioning capabilities of the four ambiguity resolution schemes are now verified with real GNSS data for dual-frequency combined GPS+BDS. The east, north, and up positioning errors as well as the ratio of fixed ambiguities $n_{\text {fixed }} / n$ are shown for 45 min of data in Figure 3 for the two FAR schemes and in Figure 4 for the PAR counterparts. The positioning errors are the difference between the estimated position and precise reference coordinates. With IB FAR, 71 epochs of data are required until

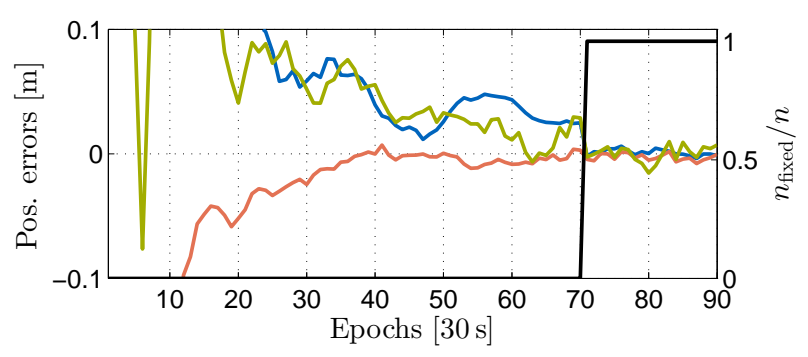

(a) IB FAR

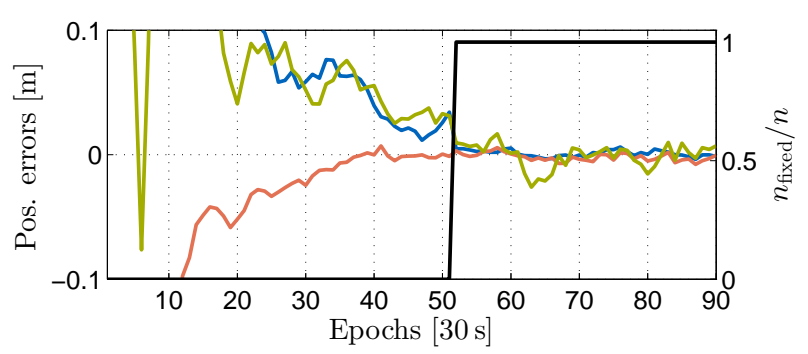

(b) DT FAR

Figure 3: East (blue), north (orange), and up (green) positioning errors with FAR for dual-frequency combined GPS+BDS RTK on PERT/NNOR with a fixed failure rate of $P_{\mathrm{f}}=0.1 \%$. The ratio of fixed ambiguities is shown in black. 


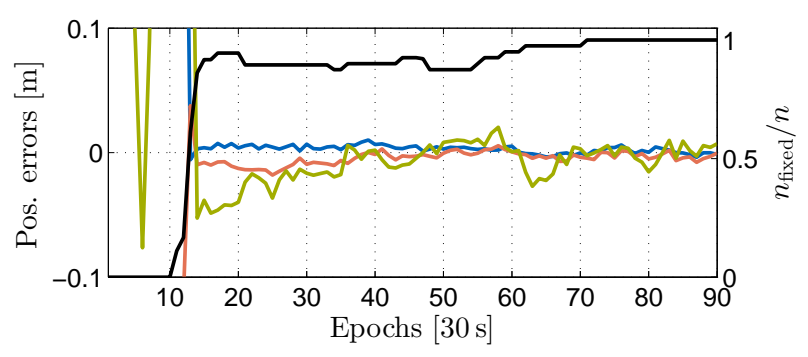

(a) IB PAR

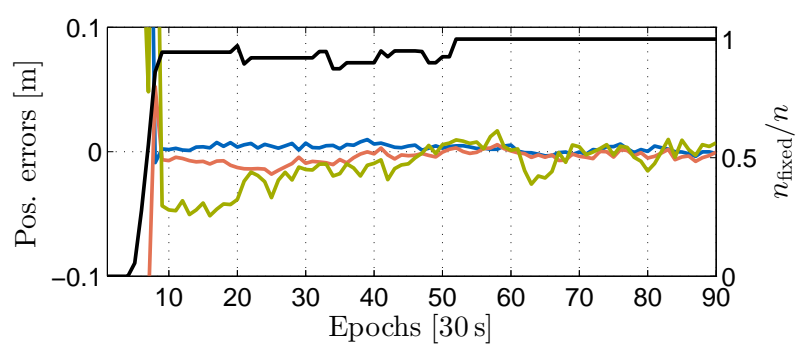

(b) DT PAR

Figure 4: East (blue), north (orange), and up (green) positioning errors with PAR for dual-frequency combined GPS+BDS RTK on PERT/NNOR with a fixed failure rate of $P_{\mathrm{f}}=0.1 \%$. The ratio of fixed ambiguities is shown in black.

the IB failure rate drops to less than $P_{\mathrm{f}}=0.1 \%$, i.e., until the full set of integer ambiguities is resolved and the fixed solution can be accepted. With the data-driven DT FAR, the ambiguities can already be reliably resolved after 52 epochs. With IB PAR, the first ambiguities are resolved after 11 epochs, and after 14 epochs the estimates of the horizontal position components are already at centimeter level, although the full set of ambiguities is again only resolved after 71 epochs. An even faster solution is obtained with DT PAR, for which the horizontal positioning error is at centimeter level after only 9 epochs. It is noted that the large numbers of epochs required by the FAR schemes are to some extend also caused by rising satellites after epoch 20 and 34 . The PAR schemes are almost not affected by this. Although the ratio of fixed ambiguities is slightly smaller immediately after the new satellite has been included, the positioning precision is not visibly reduced.

The positioning results for the whole day are shown in Figure 5 for FAR and in Figure 6 for PAR, which can be read as follows. The position errors are color coded, where gray means that no ambiguity is resolved and blue means that all $n$ ambiguities are resolved. For the PAR schemes, all shades in between are possible. The ratio of resolved ambiguities is shown in the third row, and the computed formal precision $\alpha$ in the last row. The feasible precision values are limited by the float and fully fixed solutions, which are drawn as blue lines. Since we are mostly interested in the time required to reach centimeter precision, the estimator is completely reinitialized every $90 \mathrm{~min}$, as can most notably be seen at the formal float precision.

The ratio of fixed ambiguities for the FAR schemes in Figure 5 is either 0 or 1, corresponding to the gray and blue position errors, respectively. The horizontal error of the fully fixed solution is at centimeter level, whereas the gray float solution can have much larger errors, especially after reinitialization, as can be seen in the time series of the up error. The behavior after reinitialization agrees with the computed float precision values in the last row. Although the results of IB FAR and DT FAR seem to be very similar, the fixed solution was accepted in 1729 out of 2880 epochs for IB FAR and in 2011 epochs for DT FAR. This difference is visible, e.g., in the up error after the last initialization. We further notice that the fixed solution is often lost when a satellite rises, as can be seen in the last row: The computed formal precision is identical to the float precision after initialization, before it drops to the fixed precision once the ambiguities are resolved. But then quite often the float precision is observed again for a couple of epochs at a later time instance. One could of course exclude rising satellites from the ambiguity fixing, which would be nothing else than a specific implementation of PAR.

The ratio of fixed ambiguities for the PAR strategies as shown in Figure 6 is mostly close to 1, except for a few epochs after each reinitialization. Accordingly, the computed formal precision values in the last row are mostly very close to the fully fixed precision. This is verified by the position errors, where the number of epochs with large errors is significantly reduced compared to Figure 5. The best results are obtained for DT PAR, for which the gray dots are almost completely gone and only a few epochs after each initialization remain with a position error that exceeds a few centimeters. Note that the blue dots with large positioning errors reflect the precision of partially fixed solutions and do not imply wrong ambiguity estimates. The average ratio of fixed ambiguities is $91.3 \%$ for IB PAR and $94.9 \%$ for DT PAR (as compared to $60.0 \%$ and $69.8 \%$ for the FAR counterparts). We can also see that-unlike for FAR-rising satellites hardly affect the positioning performance and are thus not an issue with PAR.

\section{CONCLUSION}

The benefit of PAR over conventional FAR was demonstrated for dual-frequency long baseline single and combined GPS L1+L2 and BDS B1+B2 RTK positioning. Each a model-driven algorithm based on the IB failure rate and a data-driven algorithm based on the DT with a maximum failure rate constraint was formulated for FAR and PAR. It was shown via simulations that clearly shorter time spans can be expected to be required in order to reach centimeter level coordinate estimates when using PAR, especially for combined GPS+BDS.

These results were confirmed by analyzing one day of real GNSS data from an $88.5 \mathrm{~km}$ baseline. It was shown that it is often not required to resolve the full set of ambiguities in order to achieve close to optimal positioning precision. Two main advantages of PAR over FAR were observed: The average number of epochs until the precision of the coordinate estimates first reached 

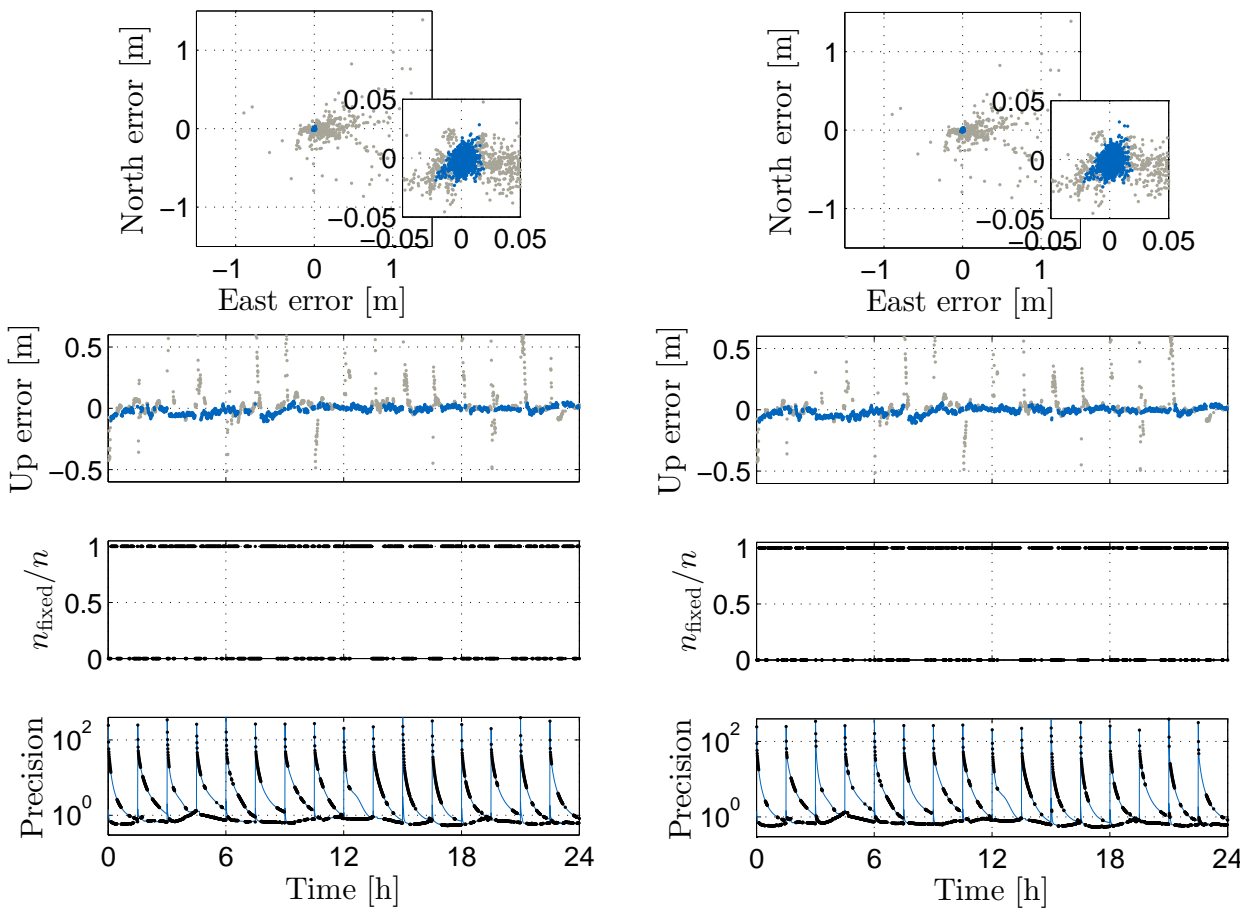

(a) IB FAR

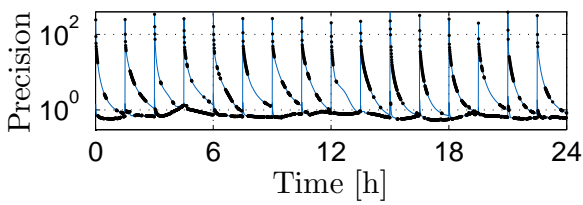

(b) DT FAR

Figure 5: Long baseline dual-frequency GPS+BDS RTK results on PERT/NNOR with FAR and a fixed failure rate of $P_{\mathrm{f}}=0.1 \%$. The color of the horizontal and vertical components of the positioning error indicates the ratio of fixed ambiguities as shown in the third row, where gray stands for a ratio of 0 and blue for a ratio of 1 . In the last row, the computed formal precision values $\alpha$ are shown in black. They are limited by the float (upper blue line) and fully fixed (lower blue line) precision. The estimator is reinitialized every $90 \mathrm{~min}$.
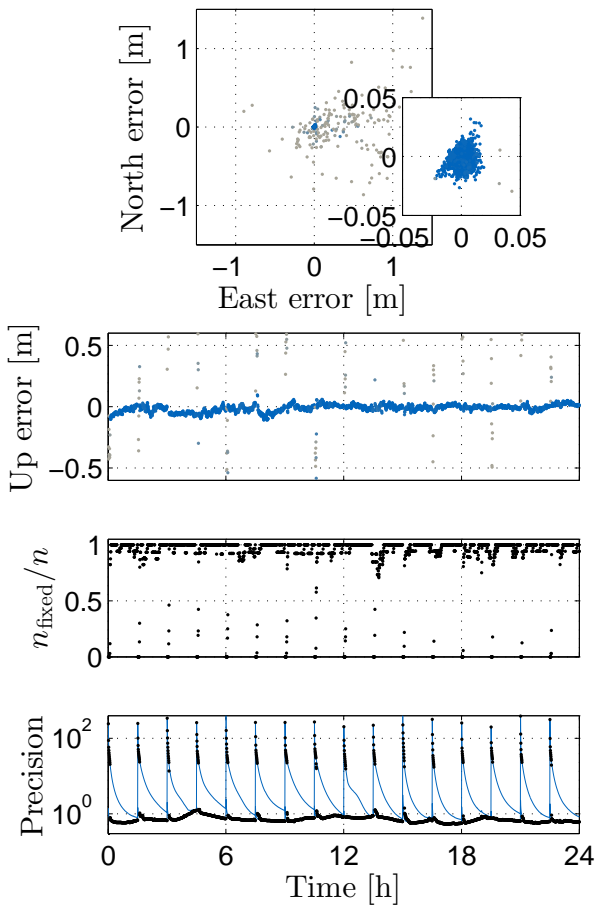

(a) IB PAR
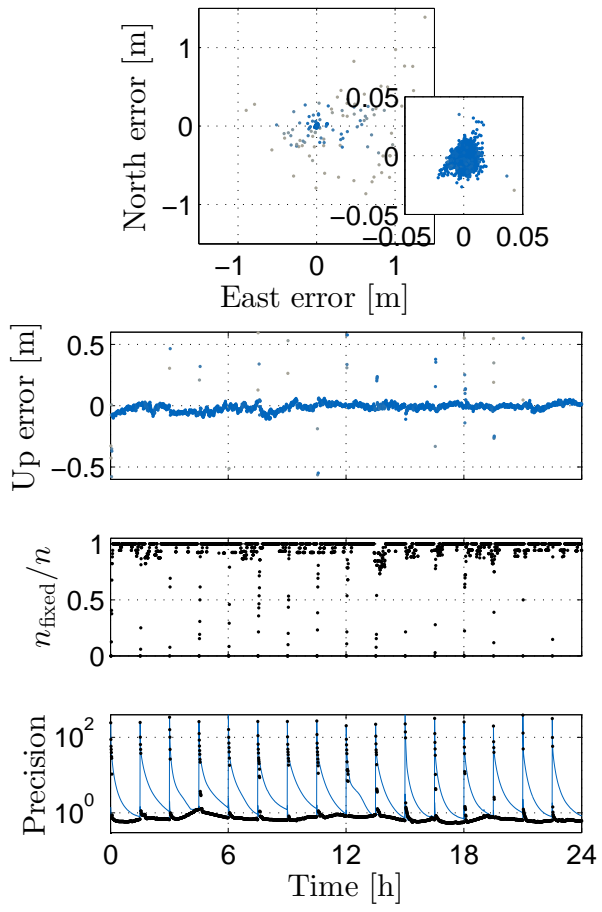

(b) DT PAR

Figure 6: Long baseline dual-frequency GPS+BDS RTK results on PERT/NNOR with PAR and a fixed failure rate of $P_{\mathrm{f}}=0.1 \%$, see caption of Figure 5. 
centimeter level was clearly reduced, and the problem of when or how to include the carrier-phase ambiguities corresponding to rising satellites in a multi-epoch positioning solution was automatically taken care of by both presented PAR strategies.

\section{ACKNOWLEDGMENTS}

The GNSS data used in this contribution was provided by the multi-GNSS experiment (MGEX) by the international GNSS service (IGS).

\section{REFERENCES}

[1] P. J. G. Teunissen and S. Verhagen, "GNSS Ambiguity Resolution: When and How to Fix or not to Fix?," in VI HotineMarussi Symposium on Theoretical and Computational Geodesy, pp. 143-148, Springer Berlin Heidelberg, 2008.

[2] S. Verhagen and P. J. G. Teunissen, "The ratio test for future GNSS ambiguity resolution," GPS Solutions, vol. 17, no. 4, pp. 535-548, 2013.

[3] D. Odijk, B. S. Arora, and P. J. G. Teunissen, "Predicting the Success Rate of Long-baseline GPS+Galileo (Partial) Ambiguity Resolution," Journal of Navigation, vol. 67, no. 3, pp. 385-401, 2014.

[4] A. Nardo, B. Li, and P. J. G. Teunissen, "Partial Ambiguity Resolution for Ground and Space-Based Applications in a GPS + Galileo Scenario: A Simulation Study," Advances in Space Research, vol. 57, no. 1, pp. 30-45, 2016.

[5] A. Brack, "Partial Ambiguity Resolution for Reliable GNSS Positioning - a Useful Tool?," in 2016 IEEE Aerospace Conference, (Big Sky, MT, USA), pp. 1-7, IEEE, 2016.

[6] C. Tiberius, T. Pany, B. Eissfeller, P. Joosten, and S. Verhagen, "0.99999999 confidence ambiguity resolution with GPS and Galileo," GPS Solutions, vol. 6, no. 1-2, pp. 96-99, 2002.

[7] C. Deng, W. Tang, J. Liu, and C. Shi, "Reliable single-epoch ambiguity resolution for short baselines using combined GPS/BeiDou system,” GPS Solutions, vol. 18, no. 3, pp. 375-386, 2014.

[8] H. He, J. Li, Y. Yang, J. Xu, H. Guo, and A. Wang, "Performance assessment of single- and dual-frequency BeiDou/GPS single-epoch kinematic positioning," GPS Solutions, vol. 18, no. 3, pp. 393-403, 2014.

[9] P. J. G. Teunissen, R. Odolinski, and D. Odijk, "Instantaneous BeiDou+ GPS RTK positioning with high cut-off elevation angles," Journal of Geodesy, vol. 88, no. 4, pp. 335-350, 2014.

[10] R. Odolinski, P. J. G. Teunissen, and D. Odijk, "Combined BDS, Galileo, QZSS and GPS single-frequency RTK," GPS Solutions, vol. 19, no. 1, pp. 151-163, 2015.

[11] R. Odolinski, P. J. G. Teunissen, and D. Odijk, "Combined GPS+BDS+Galileo+QZSS for Long Baseline RTK Positioning," in Proceedings of ION GNSS+ 2014, (Tampa, FL, USA), pp. 2326-2340, 2014.

[12] R. Odolinski, P. J. G. Teunissen, and D. Odijk, "Combined GPS + BDS for short to long baseline RTK positioning," Measurement Science and Technology, vol. 26, no. 4, p. 45801, 2015.

[13] A. Brack, "Reliable GPS+BDS RTK positioning with partial ambiguity resolution," GPS Solutions, pp. 1-10, 2016. DOI: 10.1007/s10291-016-0594-1.

[14] S. Jazaeri, A. Amiri-Simkooei, and M. A. Sharifi, "On lattice reduction algorithms for solving weighted integer least squares problems: comparative study," GPS Solutions, vol. 18, no. 1, pp. 105-114, 2014.

[15] P. J. G. Teunissen, "Integer aperture GNSS ambiguity resolution,” Artificial Satellites, vol. 38, no. 3, pp. 79-88, 2003.

[16] A. Brack and C. Günther, "Generalized integer aperture estimation for partial GNSS ambiguity fixing," Journal of Geodesy, vol. 88, no. 5, pp. 479-490, 2014.

[17] P. J. G. Teunissen, "Success probability of integer GPS ambiguity rounding and bootstrapping," Journal of Geodesy, vol. 72, no. 10, pp. 606-612, 1998.

[18] Y. Feng and J. Wang, "Computed success rates of various carrier phase integer estimation solutions and their comparison with statistical success rates," Journal of Geodesy, vol. 85, no. 2, pp. 93-103, 2011. 
[19] S. Verhagen, B. Li, and P. J. G. Teunissen, "Ps-LAMBDA: Ambiguity success rate evaluation software for interferometric applications," Computers \& Geosciences, vol. 54, pp. 361-376, 2013.

[20] A. Brack, "Optimal estimation of a subset of integers with application to GNSS," Artificial Satellites, vol. 51, no. 4, pp. 123$134,2016$.

[21] P. J. G. Teunissen, P. Joosten, and C. C. J. M. Tiberius, "Geometry-free ambiguity success rates in case of partial fixing," in Proceedings of ION NTM 1999, pp. 201-207, 1999.

[22] S. Khanafseh and B. Pervan, "New Approach for Calculating Position Domain Integrity Risk for Cycle Resolution in Carrier Phase Navigation Systems,” Aerospace and Electronic Systems, IEEE Transactions on, vol. 46, no. 1, pp. 296-307, 2010.

[23] S. Verhagen, P. J. G. Teunissen, H. van der Marel, and B. Li, "GNSS ambiguity resolution: which subset to fix," in Proceedings of IGNSS Symposium, (Sydney, Australia), 2011.

[24] C. C. J. M. Tiberius and P. J. De Jonge, "Fast Positioning Using the LAMBDA Method," in Proceedings of DSNS-95, (Bergen, Norway), 1995.

[25] H. J. Euler and C. C. Goad, "On optimal filtering of GPS dual frequency observations without using orbit information," Bulletin Géodésique, vol. 65, no. 2, pp. 130-143, 1991.

[26] MOPS, "Minimum operational performance standards for global positioning system/wide area augmentation system airborne equipment," RTCA Inc. Document No. RTCA/DO-229B, 1999.

[27] A. E. Niell, "Global mapping functions for the atmosphere delay at radio wavelengths," Journal of Geophysical Research, vol. 101, pp. 3227-3246, Feb. 1996.

[28] P. J. G. Teunissen, “The least-squares ambiguity decorrelation adjustment: a method for fast GPS integer ambiguity estimation," Journal of Geodesy, vol. 70, no. 1-2, pp. 65-82, 1995.

[29] A. Brack, “On reliable data-driven partial GNSS ambiguity resolution,” GPS Solutions, vol. 19, no. 3, pp. 411-422, 2015. 